\title{
EUVE OBSERVATIONS OF VW HYI IN SUPEROUTBURST
}

\author{
CHRISTOPHER W. MAUCHE \\ Lawrence Livermore National Laboratory, \\ L-41, P.O. Box 808, Livermore, CA 94550, USA
}

\begin{abstract}
EUVE observed the SU UMa-type dwarf nova VW Hyi in superoutburst for an interval of nearly $2 \mathrm{~d}$ in 1994 June and produced EUV light curves and the first EUV spectrum of this important CV.
\end{abstract}

\section{Observations}

VW Hyi was observed in superoutburst with EUVE from 1994 June 2.13 to 4.10 UT (RJD 9505.63 to 9507.60 ; RJD = JD - 2440000 ). The observations took place $\approx 3.5 \mathrm{~d}$ after the rise of the optical flux on $\approx$ May 29.5 $\mathrm{UT}$ and $\approx 2 \mathrm{~d}$ after the peak of the optical flux at $V \approx 8.5 \mathrm{mag}$ on $\approx$ May 31.0 UT. The Deep Survey photometer $(70 \ldots 170 \AA)$ collected data for less than one day before being shut off because of the high count rate $(\sim 20$ counts $\mathrm{s}^{-1}$ ) induced in the detector by source photons. Earth occultations and standard settings on the pipeline reduction software presently limits the useful amount of spectrometer data to 4280,4177 , and $1075 \mathrm{~s}$ for the SW $(70 \ldots 180 \AA)$, MW $(145 \ldots 370 \AA)$, and LW $(290 \ldots 760 \AA)$ spectrometers, respectively.

As measured by the Deep Survey photometer and the spectrometers, the EUV flux of VW Hyi was highly variable during the observations. The upper panel of Fig. 1 shows the count rates recorded by the SW, MW, and LW spectrometers in the wavebands $80 \ldots 170,170 \ldots 300$, and $300 \ldots 400 \AA$, respectively. Whereas the visual flux fell steadily from $V \approx 8.7 \mathrm{mag}$ to $V \approx 8.9 \mathrm{mag}$ during the observations, the EUV flux is seen to increase. On shorter time-scales, the EUV flux variations, in particular the flux diminutions, are accompanied by distinct spectral variations. The lower panel of Fig. 1 shows the ratio of the count rates in the $80 \ldots 170 \AA$ to $170 \ldots 300 \AA$ wavebands recorded by the SW and $\mathrm{MW}$ spectrometers as a function of time. Each diminution of the EUV flux is accompanied by a softening of

A. Evans and J. H. Wood (eds.), Cataclysmic Variables and Related Objects, 243-246. () 1996 Kluwer Academic Publishers. Printed in the Netherlands. 


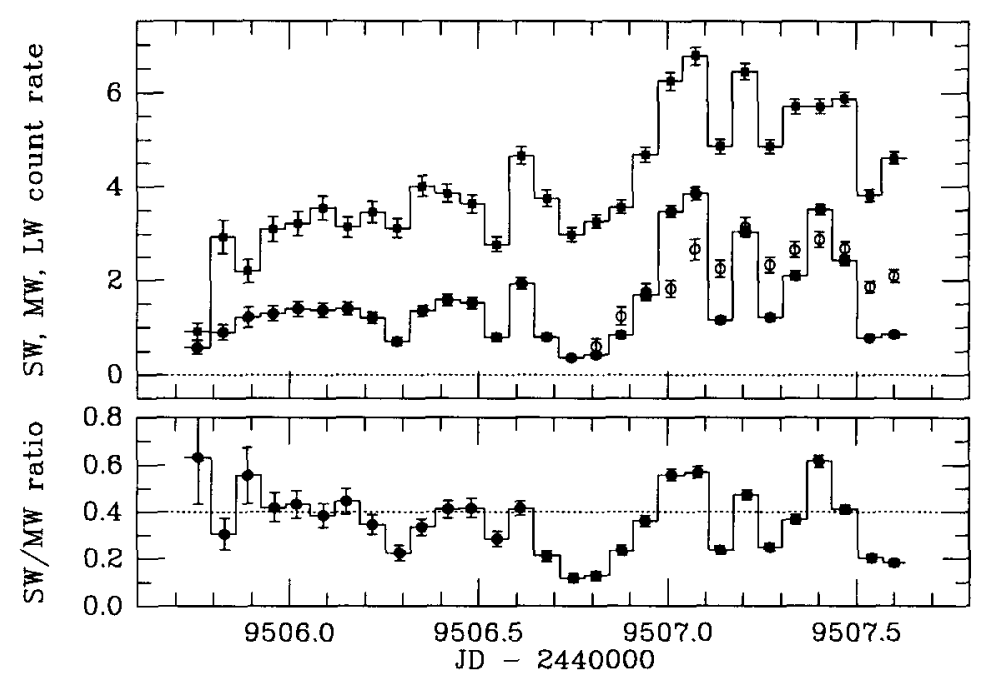

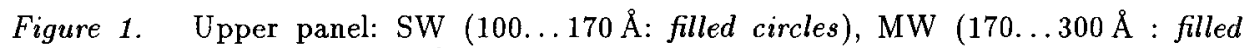
squares), and LW (300...400 $\AA$ : open circles) count rate light curves of VW Hyi. Lower panel: SW over MW (hard over soft) count rate ratio.

the spectrum; just the reverse of the effect produced by variations in the column density.

Blithely ignoring these indications that the EUV spectrum of VW Hyi varied both in flux and shape during the observations, we constructed a net spectrum for the observation using data from each of the spectrometers. The result is shown in Fig. 2. The SW, MW and LW portions of the spectrum extend from $80 \ldots 180,160 \ldots 360$ and $300 \ldots 420 \AA$, respectively, and are binned to 1,2 and $4 \AA$, respectively; approximately twice the FWHM of the resolution of each spectrometer. There is residual signal in the LW spectrometer longward even of $420 \AA$, but this signal is most likely due to second-order flux; this effect is being investigated. The errors in the flux density due solely to counting statistics are everywhere less than $110^{-12} \mathrm{erg} \mathrm{cm}^{-2} \mathrm{~s}^{-1} \AA^{-1}$, and are typically less than half this amount.

\section{Discussion}

After years of speculation about its nature (e.g. van der Woerd, Heise \& Bateson 1986; Pringle et al. 1987; Mauche et al. 1991; van Teeseling, Verbunt \& Heise 1993), it is gratifying finally to see the EUV spectrum of VW Hyi. Many aspects of the spectrum are consistent with previous measurements. The fact that the spectrum peaks longward of $170 \AA$ is con- 


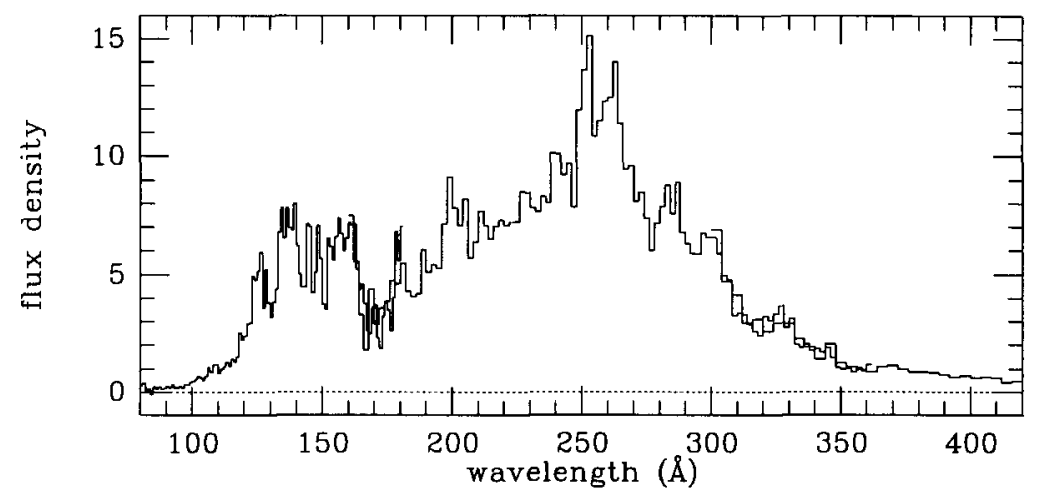

Figure 2. Net SW $(80 \ldots 180 \AA), \mathrm{MW}(160 \ldots 360 \AA)$ and LW $(300 \ldots 420 \AA)$ spectra of VW Hyi. Units of flux density are $10^{-12} \mathrm{erg} \mathrm{cm}^{-2} \mathrm{~s}^{-1} \AA^{-1}$.

sistent with the fact that, in outburst, the EXOSAT Al-Par filter count rate is higher than the 3000 Lex or 4000 Lex count rates. That the spectrum extends to yet longer wavelengths is consistent with the low neutral hydrogen column density $\left(\approx 610^{17} \mathrm{~cm}^{-2}\right)$ measured by Polidan, Mauche $\&$ Wade (1990). That the spectrum does not extend shortward of $\approx 100 \AA$ $(\approx 0.12 \mathrm{keV})$ is consistent with the non-detection in the $0.18 \ldots 0.43 \mathrm{keV}$ bandpass by HEAO-1 (Córdova et al. 1980) and with the decrease during outburst of the flux in the $0.1 \ldots 2.4 \mathrm{keV}$ bandpass measured by ROSAT (Wheatley et al. 1996). The brightness of the spectrum is inconsistent. The observed $80 \ldots 420 \AA$ flux is $1.510^{-9} \mathrm{erg} \mathrm{cm}^{-2} \mathrm{~s}^{-1}$, implying a luminosity of $7.710^{32}(d / 65 \mathrm{pc})^{2} \mathrm{erg} \mathrm{s}^{-1}$. For comparison, the luminosity of the accretion disk is $\approx 20$ times higher (Mauche et al. 1991). The predicted EXOSAT Al-Par and 3000 Lex filter count rates are 28.3 and 15.5 counts s $^{-1}$, respectively. The highest measured EXOSAT Al-Par and 3000 Lex filter count rates appear to be 4.3 and 1.2 counts $\mathrm{s}^{-1}$, respectively (van der Woerd $\&$ Heise 1987). Given the low net exposure on source, it is fortunate that VW Hyi was so bright during our observations.

It is fortunate also that the neutral hydrogen column density to VW Hyi is so low. Columns typical of other nearby CV systems $\left(N_{\mathrm{H}} \gtrsim\right.$ few $10^{19} \mathrm{~cm}^{-2}$; Mauche, Raymond \& Córdova 1988), would render VW Hyi nearly unobservable: at $N_{\mathrm{H}}=310^{19} \mathrm{~cm}^{-2}$, the optical depth of the ISM is 1.1 at $100 \AA$, 3.2 at $150 \AA, 6.5$ at $200 \AA$. SS Cyg (Mauche, Raymond \& Mattei 1995) and U Gem (Long et al. 1995) both have higher columns, but are also intrinsically harder. If other, even nearby, CVs are as soft as VW Hyi, it will be hard to impossible to detect them in the $\operatorname{EUV}(\lambda=100 \ldots 912 \AA)$.

As is the case with SS Cyg and U Gem, the EUV spectrum of VW Hyi belies simple interpretation. Consideration of the following options is in- 
structive of the problems facing us.

(i) A blackbody spectrum. To produce so little flux shortward of $\approx$ $100 \AA$, the temperature of a blackbody must be $T \lesssim 110^{5} \mathrm{~K}(k T \lesssim 10 \mathrm{eV})$. To extinguish the flux longward of $\approx 350 \AA$, the neutral hydrogen column density then must be $N_{\mathrm{H}} \gtrsim 310^{18} \mathrm{~cm}^{-2}$, but such a model fails to reproduce the overall spectral distribution.

(ii) A modified blackbody spectrum. The ionization edges of CIII-IV, N III-V, O III-IV, Ne II-IV, MgIII-IV, Si IV, S IV-VI and FeIV-VI all lie in this bandpass, but are not apparent in the spectrum. This result probably does not constrain irradiation of the white dwarf by a hard continuum spectrum, since such models produce edges at wavelengths below $100 \AA$ (van Teeseling, Heise \& Paerels 1994).

(iii) The spectrum of an optically thin plasma. At $T \approx 1$.. few $10^{5} \mathrm{~K}$, the He II Lyman lines $(\lambda=304,256,243, \ldots \AA)$ and bound-free continuum $(\lambda<228 \AA)$ are in emission, contrary to observations. At higher temperatures, particularly $T \gtrsim 510^{5} \mathrm{~K}$, the free-free continuum is weak, but strong emission lines begin to appear shortward of $100 \AA$.

Other processes likely affect the spectrum. The ISM and possibly the wind of VW Hyi will imprint a He II absorption edge at $228 \AA$ onto the intrinsic spectrum. Measurement of, or upper limits on, such an edge will provide an estimate of the integrated column density of this ion. He I has autoionization resonances at $\lambda=206,195,192, \ldots \AA$ (Rumph, Bowyer \& Vennes 1994). The first such resonance may be responsible for the emission/absorption feature at $206 \AA$.

Acknowledgements. The author is pleased to acknowledge the contributions to this research by the members, staff, and director, J. Mattei, of the AAVSO. This work was performed under the auspices of the U.S. Department of Energy by Lawrence Livermore Nat'l Laboratory under contract No. W-7405-Eng-48.

\section{References}

Córdova, F.A., Nugent, J.J., Klein, S.R., Garmire, G.P., 1980, MNRAS, 190, 87

Long, K.S., Mauche, C.W., Szkody, P., Mattei, J.A., 1995, in "Cataclysmic Variables", eds A. Bianchini et al., Kluwer, p133

Mauche, C.W., Raymond, J.C., Córdova, F.A., 1988, Ap. J., 335, 829

Mauche, C.W., Raymond, J.C., Mattei, J.A., 1995, Ap. J., 446, 842

Mauche, C. W., 1991, Ap. J., 372, 659

Polidan, R.S., Mauche, C.W., Wade, R.A., 1990, Ap. J., 356, 211

Pringle, J.E., Bateson, F.M., Hassall, B.J.M., et al., 1987, MNRAS, 225, 73

Rumph, T., Bowyer, S., Vennes, S., 1994, AJ, 107, 2108

van der Woerd, H., Heise, J., 1987, MNRAS, 225, 141

van der Woerd, H., Heise, J., Bateson, F., 1986, A\&A, 156, 252

van Teeseling, A., Heise, J., Paerels, F., 1994, A\&A, 281, 119

van Teeseling, A., Verbunt, F., Heise, J., 1993, A\&A, 270, 159

Wheatley, P.J., Verunt, F., Belloni, T., et al., 1996, A\&A, in press. 